\title{
Performance Evaluation of Token Bucket based Congestion Control Protocol for Wireless Sensor Networks
}

\author{
Harpreet Singh \\ M.Tech Research Scholar, \\ Dept. of CSE \\ Amritsar College of Engineering and Technology
}

\author{
Tanu Preet Singh \\ Professor and HOD, \\ Dept. of ECE \\ Amritsar College of Engineering and Technology
}

\begin{abstract}
The distinctive characteristics of WSN such as for instance coherent nature of traffic to base station that happens through its many-to-one topology and collision in physical channel are main factors behind congestion in wireless sensor networks. Also when sensor nodes inject sensory data into network the congestion is possible. Congestion affects the continuous flow of data, loss of information, delay in the arrival of data to the destination and undesirable usage of large amount of energy in the nodes. Therefore Congestion in wireless sensor networks (WSN) must be controlled to be able to prolong system lifetime, improve fairness, high energy-efficiency, and improve quality of service (QoS). In this paper, main focus is on improving the PASCCC further by utilizing token bucket algorithm in place of priority queues. This work in addition has dedicated to the inter cluster data aggregation to boost the outcome further. The objective is to stop congestion utilizing the token bucket algorithm.
\end{abstract}

\section{Keywords}

WSNs, congestion control, token bucket, PASCCC

\section{INTRODUCTION}

Wireless sensor networks (WSNs) are often made up of one or more sinks and tens or 1000 s of sensor nodes scattered in a real space. With integration of information sensing, computation, and wireless communication, the sensor nodes can sense physical information, process crude information, and report required information to the sink. These sensors are small, with restricted processing and computing resources. These sensor nodes can sense, measure, and gather information from the surroundings and, centered on some local decision process, they could transmit the sensed data to the user. The normal task of sensor node is to get the information from the scene of event and send the information to sink. Wireless sensor network (WSN) is really a high level of cross-disciplinary, highly integrated knowledge on network communication, and a forefront research spot in the world.

\section{CONGESTION IN WIRELESS SENSOR NETWORKS}

Several wireless sensor network applications need that the observations or findings gathered by sensors be kept at some central location. Congestion may arise while gathering the information and giving it towards the central spot in WSN. Congestion occurs mostly in the sensors-to-sink path when packets are transferred in a many-to-one manner. Congestion in WSNs has bad influence on system performance i.e., indiscriminate packet loss, increased delay in packet transfer, lost node energy and significant fidelity degradation. The objective of WSN congestion control is to improve the network throughput, lower the time of information transmitted delay. Under these conditions, node energy, communications bandwidth, network processing capacity and other resources are typically limited. It's probable to boost the network performance through the protocols design, path algorithm select, data integration and load balancing.

\section{OUTLINE OF CONGESTION CONTROL PROTOCOLS IN WIRELESS SENSOR NETWORKS}

There were several attempts to fix the issue of congestion control. In analysis of related works in the context of wireless sensor networks congestion control is presented.

\subsection{Congestion Avoidance and Detection (CODA)}

CODA is energy efficient crowding control mechanism made for WSNs. CODA, detects the congestion by noting the buffer size of sensor nodes and the load of the wireless channel. If both of these characteristic exceed from the pre-defined threshold, a sensor node notifies its neighbor to decrease the transmission rate. Before transmitting a packet, a sensor node divides the channel to fixed periods. When it found the channel hectic than pre-defined times, it adjusts a control bit to tell the base station of the congestion.

\subsection{Congestion Control and Fairness (CCF)}

$\mathrm{CCF}$ finds obstruction centered on packet service time at MAC layer and gets a grip on obstruction predicated on hopby-hop fashion with easy fairness. CCF employs packets service time to realize the available service rate and find the congestion in each node. Once the obstruction is seen, it informs the downstream nodes to lessen their data transmission rate.

\subsection{SenTCP}

SenTCP is definitely an open-loop hop-by-hop obstruction control protocol with two particular characteristics: 1) it jointly employs average regional packet service time and regional packet inter-arrival time to be able to calculate regional obstruction level in each sensor node. 2) It employs hop-by-hop obstruction control. In SenTCP, each intermediate indicator node can produce feedback signal backward and hop-by-hop. The feedback signal, which holds regional obstruction degree and the buffer occupancy proportion, is 
useful for the neighboring indicator nodes to modify their giving rate in the transport layer. Utilization of hop-by-hop feedback control may eliminate congestion easily and lower packet losing, which conserves energy. SenTCP grasps larger throughput and excellent energy-efficiency as it certainly decreases packet losing. But SenTCP copes with just obstruction and assures no consistency.

\subsection{Fairness Aware Congestion Control (FACC)}

FACC is an obstruction control process, which regulates the obstruction and defines good bandwidth allocation for every single movement of data. FACC finds the obstruction centered on packet drop rate at the base station. In FACC nodes are split into 2 types near base station and near source node centered on the site in WSNs. Each time a packet is missing, then near base station nodes deliver a Warning Message (WM) to the near source node. Following obtaining WM the near source nodes deliver a Control Message (CM) to the source node. The source nodes regulate their sending rate on the basis of the traffic on the channel and the existing sending rate. Afterwards obtaining CM, flow rate will be modified centered on just determined sending rate.

\subsection{Priority Based Congestion Control Protocol (PCCP)}

PCCP is an obstruction control process centered on node priority index that's presented to reveal the significance of each indicator node. Nodes are given a priority on the basis of the function they accomplish and their location. Nodes nearby the base station have an increased priority. The obstruction is found on the basis of the proportion of sending rate to the packet arrival rate. If the giving rate is leaner, it suggests that obstruction has occurred. The obstruction data is piggybacked in information packet header combined with priority index. Nodes regulate their giving rate with regard to the obstruction at the node itself. PCCP attempts to lessen packet loss in obstruction state while reaching the measured equity transmission for single-path and multipath routing.

\section{LITERATURE SURVEY}

Watfa, Mohamed K. et al. [1] provided an on the web mathematical battery model suitable for implementation in sensor networks. Using our battery model, we propose a new Battery Aware Reliable Clustering (BARC) algorithm for WSNs. BARC incorporates many features which are missing in many other clustering algorithms. It rotates cluster heads $(\mathrm{CHs})$ according to a battery recovery scheme and it also incorporates a trust factor for selecting cluster heads thus increasing reliability. Most importantly, our proposed algorithm relaxes most of the rigid assumptions that the other algorithms impose such as the ability of the cluster head to communicate directly with the base station and having a fixed communication radius for intra-cluster communication. BARC uses Z-MAC which has several advantages over other MAC protocols. Simulation results reveal that using BARC prolongs the network lifetime greatly in comparison to when compared with other clustering techniques. Misra, Sudip, and P. Dias Thomasinous [2] discussed that the area of wireless sensor networks (WSN) happens to be attractive in the research community area due to its applications in diverse fields such as defense security, civilian applications and medical research. Routing is a serious issue in WSN due to the use of computationally-constrained and resourceconstrained micro-sensors. These constraints prohibit the deployment of traditional routing protocols designed for other ad hoc wireless networks. Any routing protocol designed for use in WSN should be reliable, energy-efficient and should increase the lifetime of the network. They proposed a simple, least-time, energy-efficient routing protocol with one-level data aggregation that ensures increased life time for the network. It was observed that the proposed protocol outperformed them in throughput, latency, average energy consumption and average network lifetime. The proposed protocol uses absolute time and node energy as the criteria for routing, this ensures reliability and congestion avoidance. Saleem, Muhammad et al. [3] proposed beeinspired BeeSensor protocol that is energy-aware, scalable and efficient. The important contribution of this work is a three phase protocol design strategy: (1) we first take inspiration from biological systems to develop a distributed, decentralized and simple routing protocol, (2) we formally model important performance metrics of our protocol to get an analytic insight into its behavior, and (3) we improve our protocol on the basis of our analysis in phase 2 . We then evaluate its performance in a sensor network simulator. The results of our experiments demonstrate the utility of this three phase protocol engineering, which helped BeeSensor in achieving the best performance with the least communication and processing costs - two main sources of energy consumption in sensor networks - as compared to other SI based WSN routing protocols. Taheri, Hoda et al. [4] proposed an energy-aware distributed dynamic clustering protocol (ECPF) which applies three techniques: (1) nonprobabilistic cluster head ( $\mathrm{CH}$ ) elections, (2) fuzzy logic, and (3) on demand clustering. The remaining energy of the nodes is the primary parameter for electing tentative $\mathrm{CHs}$ using a non-probabilistic fashion. A non-probabilistic $\mathrm{CH}$ election is implemented by introducing a delay inversely proportional to the residual energy of each node. Therefore, tentative $\mathrm{CHs}$ are selected based on their remaining energy. In addition, fuzzy logic is employed to evaluate the fitness (cost) of a node in order to choose a final $\mathrm{CH}$ from the set of neighboring tentative CHs. On the other hand, every regular (non $\mathrm{CH}$ ) node elects to connect to the $\mathrm{CH}$ with minimal fuzzy cost in its neighborhood. Besides, in ECPF, $\mathrm{CH}$ elections are performed sporadically (in contrast to performing it every round). Simulation results demonstrate that our approach performs much better than protocols (LEACH, HEED, and CHEF) in terms of extending network lifetime and saving energy. Zungeru, Adamu Murtala et al. [5] presented a comprehensive survey and comparison of routing protocols in WSNs. The first part of the paper surveys state-of-the-art routing protocols in WSNs from classical routing protocols to swarm intelligence based protocols. The routing protocols are categorized based on their computational complexity, network structure, energy efficiency and path establishment. The second part of the main paper presents a comparison of a representative number of classical and swarm based protocols. Comparing routing protocols in WSNs is currently a very challenging task for protocol designers. Often, much time is required to re-create and re-simulate algorithms from descriptions in published papers to perform the comparison. Compounding the problem is that some simulation parameters and performance metrics may possibly not be mentioned. We see a need in the research community to have standard simulation and performance metrics for comparing different protocols. To this end, the final part of the paper re-simulates different protocols using a Matlab based simulator: routing modeling application simulation environment (RMASE), and gives simulation results for standard simulation and performance metrics which hopefully will serve as a 
benchmark for future comparisons for the research community. Antoniou, Pavlos et al. [6] proposed that the flocking behavior of birds can guide the design of a robust, scalable and self-adaptive congestion control protocol in the context of wireless sensor networks (WSNs). The proposed approach adopts a swarm intelligence paradigm inspired by the collective behavior of bird flocks. The main idea is to 'guide' packets (birds) to form flocks and flow towards the sink (global attractor), whilst trying to avoid congestion regions (obstacles). The direction of motion of a packet flock is influenced by repulsion and attraction forces between packets, as well as the field of view and the artificial magnetic field in the direction of the artificial magnetic pole (sink). The proposed approach is simple to implement at the individual node, involving minimal information exchange. In addition, it displays global self- $*$ properties and emergent behavior, achieved collectively without explicitly programming these properties into individual packets. Performance evaluations show the effectiveness of the proposed Flock-based Congestion Control (Flock-CC) mechanism in dynamically balancing the offered load by effectively exploiting available network resources and moving packets to the sink. Furthermore, Flock-CC provides graceful performance degradation in terms of packet delivery ratio, packet loss, delay and energy tax under low, high and extreme traffic loads. In addition, the proposed approach achieves robustness against failing nodes, scalability in different network sizes and outperforms typical conventional approaches. Zin, Shazana Md et al. [7] reviewed the state-of-the-art for secure WSN routing protocols that illustrates the issues and challenges in the context design matters. Further, we propose the schematic taxonomy of key design issues for WSN routing protocols. We also define design factors categorization relevant to secure routing: basic, essential, and optional. The similarities and differences of secure routing approaches are summarized on the basis of key design attributes, security objectives, and attacks prevention. Finally, we outline possible future research trends on secure routing design in WSN. Adhikari, Ratnadip et al. [8] have found prolific applications in a wide range of domains which include mobile phone monitoring, robotic exploration, disaster management, intrusion detection and medical systems. Medium Access Control (MAC) protocols constitute an important group of regulations which enables the successful and smooth operation of the WSN. A fundamental design goal of all MAC protocols is to prevent energy wastes from various possible sources during data communications. Till now, a wide variety of MAC protocols with different objectives have been accumulated in sensor network literature. A thorough study of these protocols is very important both from the perspectives of understanding the existing research trends and determining scopes for further innovative works in this domain. This paper meticulously discusses about the associated issues and difficulties which are faced in designing efficient MAC protocols for WSNs. Several popular MAC protocols are described here with their inherent merits and demerits. In order to provide an up-to-date survey, various MAC protocols which have been developed relatively recently are discussed, together with the traditional benchmark ones. Finally, this paper concludes with outlining a number of innovative ideas and future research directions in this domain. Mohanty, Prabhudutta, and Manas Ranjan Kabat [9] presented a Hierarchical Energy Efficient Reliable Transport Protocol (HEERTP) for the data transmission within the WSN. This protocol maximizes the network lifetime by controlling the redundant data transmission with the co-ordination of Base Station (BS). The proposed protocol also achieves end-to-end reliability using a hop-by-hop acknowledgement scheme. We evaluate the performance of the proposed protocol through simulation. The simulation results reveal that our proposed protocol achieves better performance in terms of energy efficiency, latency and reliability than the existing protocols. Jan, Mian Ahmad et al. [10] proposed a priority-based application-specific congestion control clustering (PASCCC) protocol, which integrates the mobility and heterogeneity of the nodes to detect congestion in a network. PASCCC decreases the duty cycle of each node by maintaining threshold levels for various applications. The transmitter of a sensor node is triggered when the reading of a specific captured event exceeds a specific threshold level. Time-critical packets are prioritized during congestion in order to maintain their timeliness requirements. In our proposed approach, $\mathrm{CHs}$ ensure coverage fidelity by prioritizing the packets of distant nodes over those of nearby nodes. A novel queue scheduling mechanism is proposed for $\mathrm{CHs}$ to achieve coverage fidelity, which ensures that the extra resources consumed by distant nodes are utilized effectively. The effectiveness of PASCCC was evaluated based on comparisons with existing clustering protocols. The experimental results demonstrated that PASCCC achieved better performance in terms of the network lifetime, energy consumption, data transmission, and other QoS metrics compared with existing approaches. Zhang, De-gan et al. [11] proposed an Extended Ad hoc On-Demand Distance Vector (EAODV) routing method based on distributed minimum transmission (DMT) multicast routing for WSN in this paper. According to the causes of uncertain information of WSN such as random load, data fusion and other impact factors, the DMT-based EAODV routing method chooses the forwarding routes which can connect more multicast receivers to solve routing optimization problem. The method proposes a powerful strategy to change the multicast tree structure based on the characteristic of node mobility of WSN. Experimental results demonstrate that the method effectively improves the efficiency of multicast routing. The most obvious advantage and novelty of the proposed method is its good performance in package loss rate, throughput, energy consumption and delay. Tanwar, Sudeep et al. [12] have categorized various heterogeneous routing protocols for WSNs based upon various predefined parameters. Relative comparison of various protocols with other existing state-of-the-art protocols is provided with respect to various performance evaluation parameters such as- $\mathrm{CH}$ Selection, Energy Efficiency, Security, and Application Specific. The broad categorization gives insights to various users to select one of the protocols from different categories based on its merits over the others. Zin, Shazana Md et al. [13] surveyed, explored, and informed researchers regarding the landscape of multipath routing by providing the motivation behind multipath routing deployment. Subsequently, this paper analyzes the security requirements and common attacks in wireless sensor networks. We provide a classification of secure multipath routing protocols on the basis of nature of defense against the WSN attacks. According to the classification, we investigate the existing secure multipath routing protocols within the WSN domain by discussing their strengths, limitations, and efficiency analysis. A comparative study of the suggested classification is presented based upon the multipath technique, additional security infrastructure, security requirements, corresponding attacks, and efficiency in search for effective secure routing in wireless sensor networks. 


\section{PROPOSED METHODOLOGY}

Figure 1 shows the flowchart of the proposed methodology.

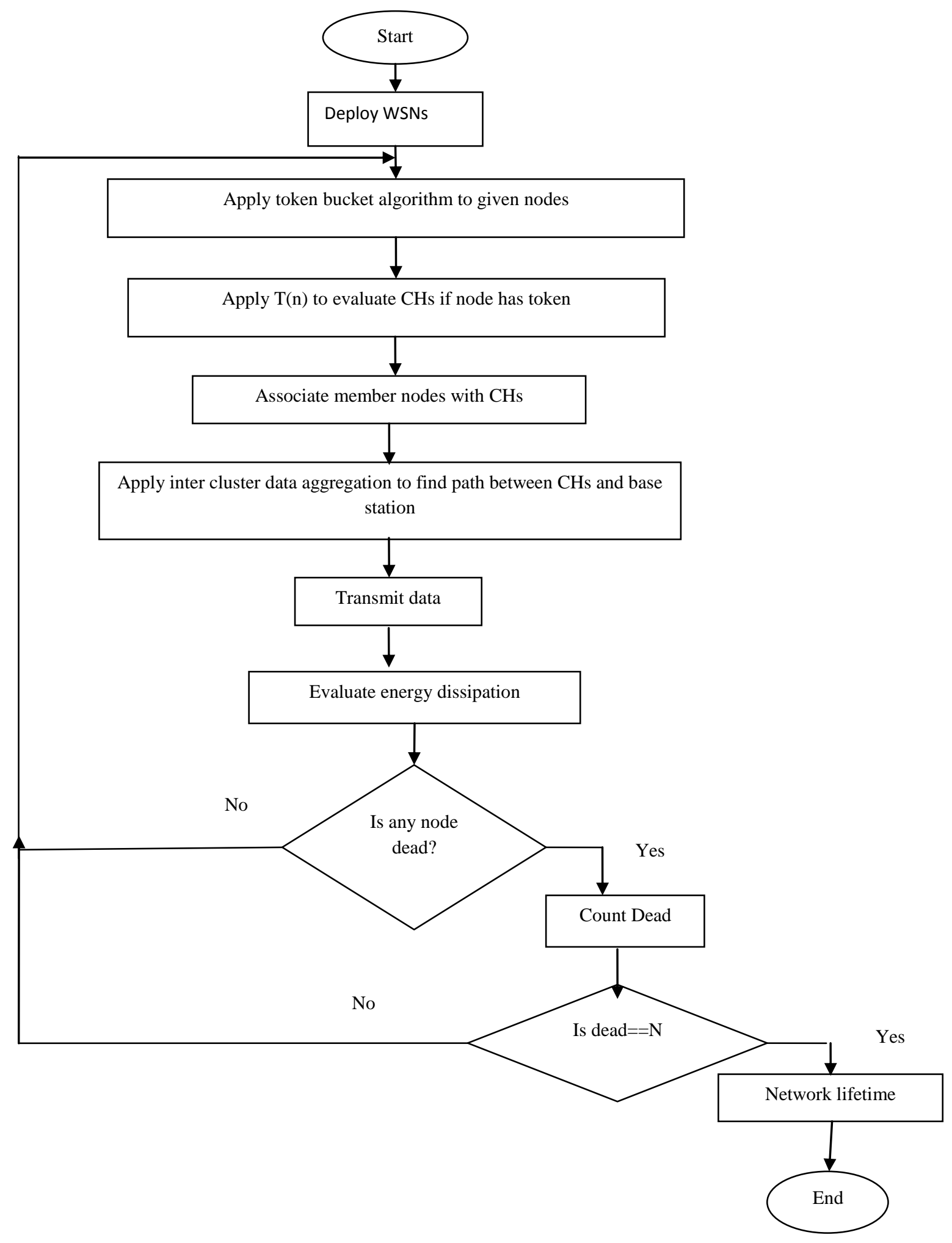

Fig 1: Flowchart of the proposed methodology 


\section{RESULTS AND DISCUSSSIONS}

\subsection{First Node Dead}

Table 1 shows the first node dead evaluation of the existing and the proposed protocols. In the table, it is clearly shown that the proposed technique performs well as compared to the existing technique.

\section{Table 1: First Node Dead Evaluation}

\begin{tabular}{|c|c|c|}
\hline Energy & $\begin{array}{c}\text { Existing } \\
\text { Technique }\end{array}$ & $\begin{array}{c}\text { Proposed } \\
\text { Technique }\end{array}$ \\
\hline 0.1 & 71 & 197 \\
\hline 0.11 & 88 & 222 \\
\hline 0.12 & 85 & 220 \\
\hline 0.13 & 94 & 253 \\
\hline 0.14 & 102 & 268 \\
\hline 0.15 & 114 & 304 \\
\hline 0.16 & 124 & 306 \\
\hline 0.17 & 142 & 345 \\
\hline 0.18 & 136 & 369 \\
\hline 0.19 & 137 & 356 \\
\hline 0.2 & 153 & 399 \\
\hline 0.21 & 170 & 434 \\
\hline 0.22 & 173 & 441 \\
\hline 0.23 & 185 & 461 \\
\hline 0.24 & 196 & 499 \\
\hline 0.25 & 186 & 513 \\
\hline 0.26 & 211 & 548 \\
\hline 0.27 & 212 & 583 \\
\hline 0.28 & 214 & 578 \\
\hline 0.29 & 229 & 605 \\
\hline 0.30 & 221 & 630 \\
\hline & & \\
\hline
\end{tabular}

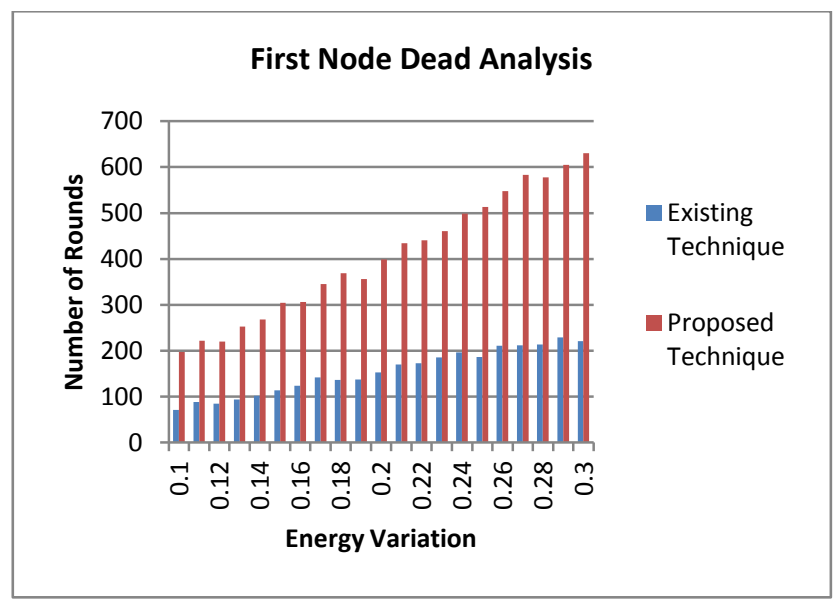

Fig 2: First Node Dead Analysis

Fig.2 is showing the comparison of existing and the proposed with respect to total number of rounds in case of first dead node. $\mathrm{X}$-axis is representing initial energy. $\mathrm{Y}$-axis is representing the number of rounds. It has been clearly shown that the overall numbers of rounds in case of proposed technique are quite more than that of the existing technique. Thus proposed technique outperforms over the existing technique.

\subsection{Half Node Dead}

Table 2 shows the half node dead evaluation of the existing and the proposed protocols. In the table, it is clearly shown that the proposed technique performs better as compared to the existing technique.
Table 2: Half Node Dead Evaluation

\begin{tabular}{|c|c|c|}
\hline Energy & $\begin{array}{c}\text { Existing } \\
\text { Technique }\end{array}$ & $\begin{array}{c}\text { Proposed } \\
\text { Technique }\end{array}$ \\
\hline 0.1 & 103 & 223 \\
\hline 0.11 & 112 & 247 \\
\hline 0.12 & 124 & 274 \\
\hline 0.13 & 135 & 301 \\
\hline 0.14 & 149 & 323 \\
\hline 0.15 & 154 & 341 \\
\hline 0.16 & 167 & 367 \\
\hline 0.17 & 180 & 394 \\
\hline 0.18 & 183 & 415 \\
\hline 0.19 & 199 & 438 \\
\hline 0.2 & 204 & 474 \\
\hline 0.21 & 216 & 491 \\
\hline 0.22 & 218 & 517 \\
\hline 0.23 & 252 & 530 \\
\hline 0.24 & 259 & 555 \\
\hline 0.25 & 250 & 584 \\
\hline 0.26 & 282 & 614 \\
\hline 0.27 & 262 & 635 \\
\hline 0.28 & 290 & 644 \\
\hline 0.29 & 295 & 671 \\
\hline 0.30 & 303 & 702 \\
\hline & & \\
\hline
\end{tabular}

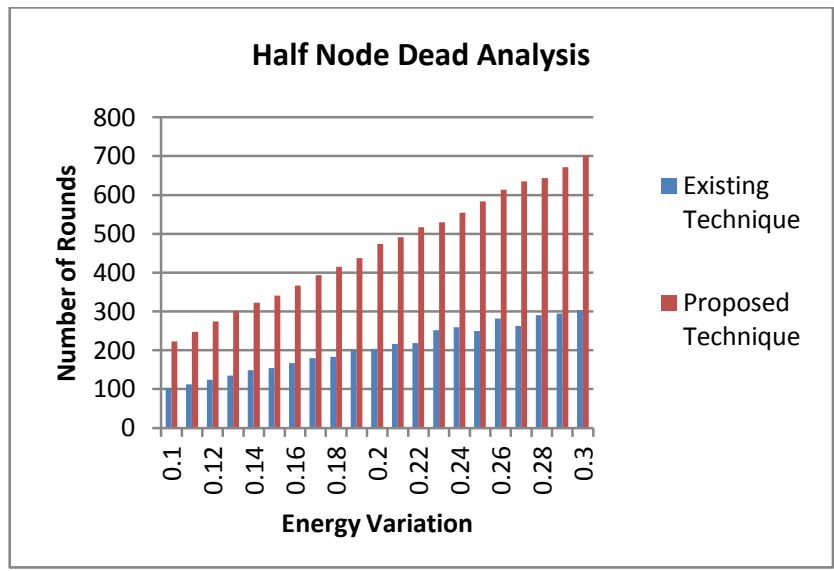

Fig 3: Half Node Dead Analysis

Fig. 3 is showing the comparison of existing and the proposed with respect to total number of rounds in case of half dead node. $\mathrm{X}$-axis is representing initial energy. Y-axis is representing the number of rounds. It has been clearly shown that the overall numbers of rounds in case of proposed technique are quite more than that of the existing technique. Thus proposed technique outperforms over the existing technique.

\subsection{All Nodes Dead}

Table 3 shows the all node dead evaluation of the existing and the proposed protocols. In the table, it is clearly shown that the proposed technique performs better as compared to the existing technique.

Table 3: All Node Dead Evaluation

\begin{tabular}{|c|c|c|}
\hline Energy & $\begin{array}{c}\text { Existing } \\
\text { Technique }\end{array}$ & $\begin{array}{c}\text { Proposed } \\
\text { Technique }\end{array}$ \\
\hline 0.1 & 280 & 319 \\
\hline 0.11 & 328 & 353 \\
\hline 0.12 & 354 & 380 \\
\hline 0.13 & 395 & 415 \\
\hline
\end{tabular}




\begin{tabular}{|c|c|c|}
\hline 0.14 & 379 & 448 \\
\hline 0.15 & 488 & 483 \\
\hline 0.16 & 462 & 506 \\
\hline 0.17 & 497 & 529 \\
\hline 0.18 & 570 & 566 \\
\hline 0.19 & 605 & 597 \\
\hline 0.2 & 569 & 617 \\
\hline 0.21 & 659 & 664 \\
\hline 0.22 & 627 & 707 \\
\hline 0.23 & 706 & 784 \\
\hline 0.24 & 732 & 753 \\
\hline 0.25 & 723 & 786 \\
\hline 0.26 & 867 & 797 \\
\hline 0.27 & 832 & 823 \\
\hline 0.28 & 798 & 867 \\
\hline 0.29 & 770 & 940 \\
\hline 0.30 & 873 & 948 \\
\hline
\end{tabular}

Fig. 4 is showing the comparison of existing and the proposed with respect to total number of rounds in case of all dead node. $\mathrm{X}$-axis is representing initial energy. Y-axis is representing the number of rounds. It has been clearly shown that the overall number of rounds in case of proposed technique is quite more than that of the existing technique. Thus proposed technique outperforms over the existing technique.

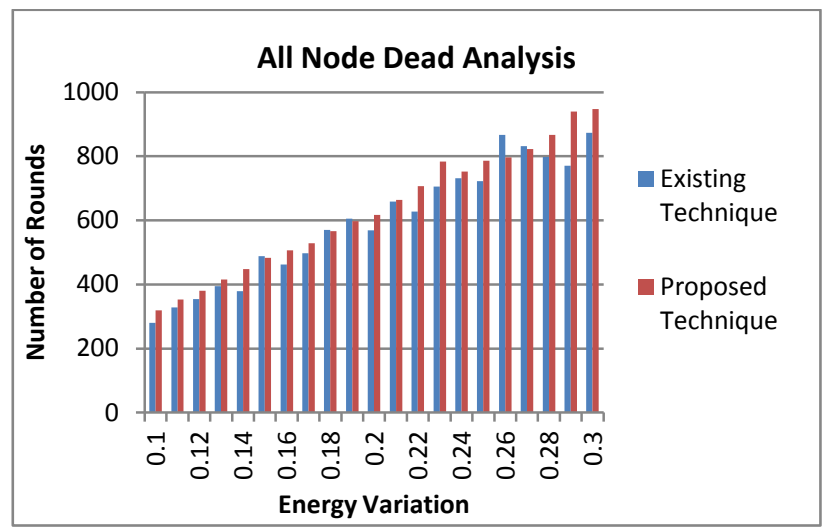

Fig 4: All Node Dead Analysis

\section{CONCLUSION AND FUTURE SCOPE}

Recently there is a huge rising fascination with Wireless Sensor Networks (WSN). The influence of WSN on our everyday living may be compared to what Internet has done to us. Both factors of obstruction control and reliability assists in lowering packet loss, which benefits in an energy efficient function of the system, which really is a critical aspect in rising the duration of sensor network. Yet another element to be taken under consideration by the transport protocols is the restricted resources of the node devices. Though these obstruction control techniques are encouraging you can find however several issues to fix in wireless sensor network to deal with obstruction efficiently. In this paper, a priority centered application specific congestion control clustering protocol has been improved following studying it since it combines the mobility and heterogeneity of the nodes to discover congestion in a network.

\section{REFERENCES}

[1] Watfa, Mohamed K., Omar Mirza, and Jad Kawtharani. "BARC: A Battery Aware Reliable Clustering algorithm for sensor networks." Journal of Network and Computer Applications 32, no. 6 (2009): 1183-1193.

[2] Misra, Sudip, and P. Dias Thomasinous. "A simple, least-time, and energy-efficient routing protocol with one-level data aggregation for wireless sensor networks." Journal of systems and software 83, no. 5 (2010): 852-860.

[3] Saleem, Muhammad, Israr Ullah, and Muddassar Farooq. "BeeSensor: An energy-efficient and scalable routing protocol for wireless sensor networks."Information Sciences 200 (2012): 38-56.

[4] Taheri, Hoda, Peyman Neamatollahi, Ossama Mohamed Younis, Shahrzad Naghibzadeh, and Mohammad Hossein Yaghmaee. "An energy-aware distributed clustering protocol in wireless sensor networks using fuzzy logic." Ad Hoc Networks 10, no. 7 (2012): 14691481.

[5] Zungeru, Adamu Murtala, Li-Minn Ang, and Kah Phooi Seng. "Classical and swarm intelligence based routing protocols for wireless sensor networks: A survey and comparison." Journal of Network and Computer Applications 35, no. 5 (2012): 1508-1536.

[6] Antoniou, Pavlos, Andreas Pitsillides, Tim Blackwell, Andries Engelbrecht, and Loizos Michael. "Congestion control in wireless sensor networks based on bird flocking behavior." Computer Networks 57, no. 5 (2013): 1167-1191.

[7] Zin, Shazana Md, Nor Badrul Anuar, Miss Laiha Mat Kiah, and Al-Sakib Khan Pathan. "Routing protocol design for secure WSN: Review and open research issues." Journal of Network and Computer Applications 41 (2014): 517-530.

[8] Adhikari, Ratnadip. "A meticulous study of various medium access control protocols for wireless sensor networks." Journal of Network and Computer Applications 41 (2014): 488-504.

[9] Mohanty, Prabhudutta, and Manas Ranjan Kabat. "A Hierarchical Energy Efficient Reliable Transport Protocol for Wireless Sensor Networks." Ain Shams Engineering Journal 5, no. 4 (2014): 1141-1155.

[10] Jan, Mian Ahmad, Priyadarsi Nanda, Xiangjian He, and Ren Ping Liu. "PASCCC: Priority-based applicationspecific congestion control clustering protocol." Computer Networks 74 (2014): 92-102.

[11] Zhang, De-gan, Xiao-dong Song, Xiang Wang, and Yuan-ye Ma. "Extended AODV routing method based on distributed minimum transmission (DMT) for WSN." AEU-International Journal of Electronics and Communications 69, no. 1 (2015): 371-381.

[12] Tanwar, Sudeep, Neeraj Kumar, and Joel JPC Rodrigues. "A systematic review on heterogeneous routing protocols for wireless sensor network." Journal of Network and Computer Applications 53 (2015): 39-56.

[13] Zin, Shazana Md, Nor Badrul Anuar, Miss Laiha Mat Kiah, and Ismail Ahmedy. "Survey of secure multipath routing protocols for WSNs." Journal of Network and Computer Applications (2015). 